\title{
Future Neutrino Oscillation Facilities
}

\section{Ilias Efthymiopoulos*}

CERN - CH 1211 Geneva 23 - Switzerland

E-mail: Ilias.Efthymiopoulos@cern.ch

\begin{abstract}
An outline of the present and future long-baseline neutrino facilities with emphasis on the possibilities at CERN is presented. Accelerator-made neutrinos for long-baseline oscillation experiments open the exploration to a broad and rather interesting field of physics experiments, with the measurement of the neutrino mixing angle $\left(\theta_{13}\right)$, the determination of the sign of neutrino mass hierarchy $\left(\Delta m_{32}^{2}\right)$ and the search for CP violation in neutrino sector as an ultimate goal. CERN presently operates the CNGS neutrino beam servicing the OPERA and ICARUS experiments at Gran Sasso aiming at the discovery of $v_{\mu}$ to $v_{\tau}$ oscillation appearance. Options for future facilities include high-intensity muon neutrino beams from pion decay (Super-Beams), electron neutrino beams from nuclei decays (Beta Beam), or muon and electron neutrino beams from muon decay (Neutrino Factory), each associated with one or several options for detector systems. Synergetic possibilities between the proposed facilities and the technical challenges for the accelerators will be discussed.
\end{abstract}

The 2011 Europhysics Conference on High Energy Physics-HEP 2011,

July 21-27, 2011

Grenoble, Rhône-Alpes France

\footnotetext{
* Speaker.
} 


\section{Introduction}

The study of neutrino oscillations is an ongoing activity since the first observation of the phenomenon through measurements using neutrinos produced in the sun or by cosmic-ray interactions in the atmosphere [1]. The experimental data on neutrino oscillations can be described assuming a unitary "mixing matrix" that rotates the mass basis (mass eigenstates) into the three observed neutrinos (or flavour eigenstates): $v_{e}, v_{\mu}, v_{\tau}$. The mixing matrix is parameterized by three mixing angles $\left(\theta_{12}, \theta_{23}, \theta_{13}\right)$ and a phase parameter $\delta$.

Present and future experimental installations using neutrinos produced by high-energy particle accelerators in the decay of pions, muons or radioactive ions, aim to unveil the theory of the neutrino oscillation, measure the parameters of the neutrino mass mixing, understand the origin of their mass - Dirac or Majorana nature, and investigate if there is CP violation in the leptonic sector. All very important physics questions that may provide hints for physics beyond the standard model and with important implications for cosmology and our understanding of the Universe.

\section{The present facilities}

At present three long-baseline accelerator neutrino facilities are in operation: the T2K beam in Japan/J-PARC [2], the NuMI beam in USA/FNAL [3] and the CNGS beam in Europe/CERN [4]. They are second generation neutrino beams, as they cope with far greater beam power than the historical first generation ones. In all cases, accelerated protons impinge a target to produce charged pions which are captured and focused using magnetic horns, and left to decay to neutrinos in a drift region. A hadron absorber is placed at the end of the decay volume followed by appropriate muon shielding prior to muon monitoring station(s) or near detector(s). The produced neutrinos continue through the earth for hundreds of kilometers to the far detector located in an underground cavern.

To detect and study the produced neutrinos at a reasonable rate, large massive detectors and a high flux of neutrinos from the beam are required, which is translated to very intense proton beams. The accelerator structure to produce these intense proton beams, along with the design and operation of the target and focusing systems, are the main challenges of these facilities. The NuMI beam at FNAL has been in operation for six years. It presently holds the beam power and intensity record, operating at $\sim 400 \mathrm{~kW}$ with a total of $1.310^{21}$ protons on target at $120 \mathrm{GeV}$, accumulated with more than 50 million extracted beam pulses. The T2K (Tokai to Kamioka) beam at J-PARC started operation in January 2010 but interrupted due to the earthquake in March 2011. In this short run period, the beam power was increased to $145 \mathrm{~kW}$ with $910^{13}$ protons per $6 \mathrm{~s}$ pulse, collecting a total of $1.4310^{20}$ protons on target. The T2K facility, being the most recent one, collects the experience from the others, with its infrastructure designed for (multi)MW of beam power as expected for future upgrades.

CNGS (CERN Neutrinos to Gran Sasso) is the European long-baseline neutrino facility. It uses the fast extracted proton beam from CERN SPS at $400 \mathrm{GeV}$ to produce intense $v_{\mu}$ beam $\left(10^{17}\right.$ $v_{\mu} /$ day) towards the Grand Sasso underground laboratory, where two large detectors OPERA and ICARUS are installed. The beam line and experiments are optimized to detect the appearance of $v_{\tau}$ neutrinos from the $v_{\mu} \rightarrow v_{\tau}$ oscillations. CNGS started operation in 2006, and its physics program since 2008 after solving successfully some initial technical issues, a proof of the chal- 

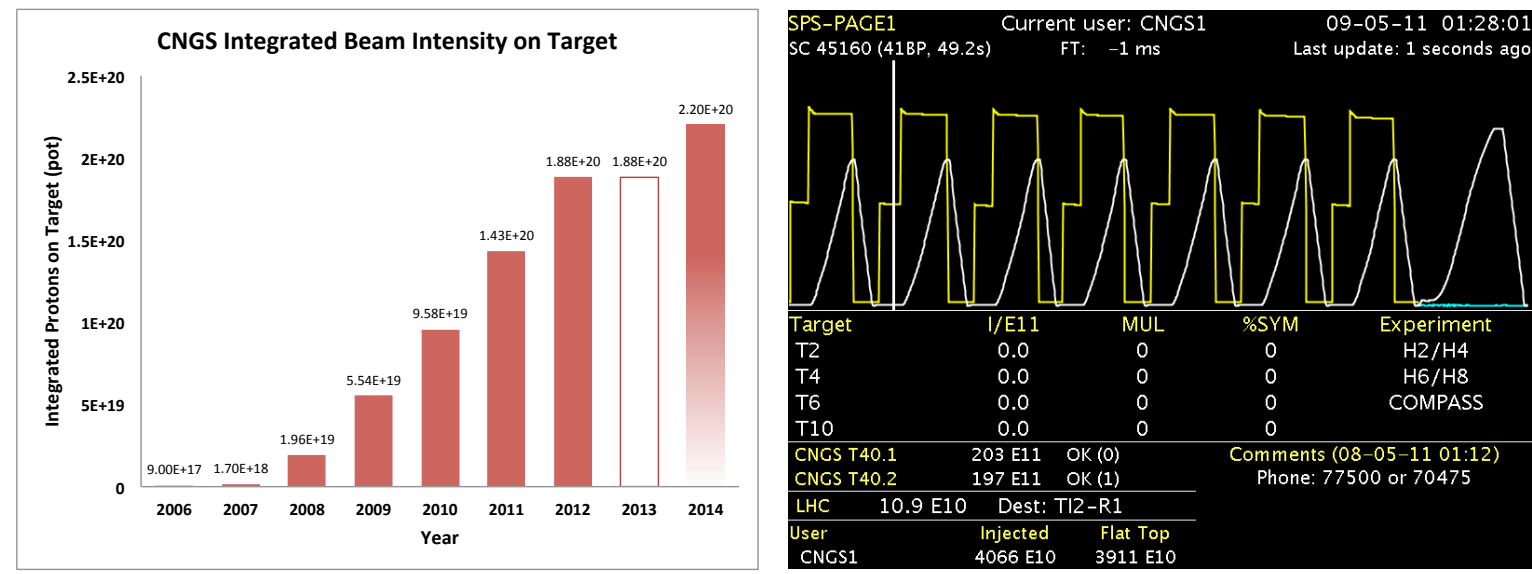

Figure 1: Left: Summary of the CNGS beam statistics and projection for the next years. Right: The SPS super cycle during operations with seven CNGS $6 \mathrm{~s}$ cycles followed by an LHC filling cycle. In this mode CNGS operates at $323 \mathrm{~kW}$ of average beam power, corresponding to $80 \%$ of the nominal beam intensity per pulse.

lenges involved. At the end of 2011 run, $1.310^{20}$ protons are delivered on target (Figure 1), that corresponds to $60 \%$ of the approved program (5 years $\times 4.510^{19}$ protons/year). The OPERA experiment observed the first $v_{\tau}$ event this year [5] and the ICARUS operates smoothly since 2010, demonstrating the power of the liquid argon technique for large volume neutrino detectors [6].

\section{The future facilities}

Upgrade scenarios of the present facilities and proposals for new long-baseline options are in preparation world-wide. For the conventional beams the road map is summarized in Figure 2. First, operation with increased beam power reaching around $700 \mathrm{~kW}$ is planned, followed by a second and more challenging step to reach the (multi)MW level. While the first stage looks achievable with today's technology and gained experience, working at the (multi)MW scale is still a major challenge and substantial R\&D is required to find technically viable solutions for the critical systems like the target, horns, beam windows and dumps.

For T2K the restart of the facility after the earthquake is foreseen for Spring 2012 aiming for a smooth increase of the beam power to reach $500 \mathrm{~kW}$ hopefully in 2013 and in a few years $750 \mathrm{~kW}$. To further continue for the design goal of $1.66 \mathrm{MW}$, upgrades of the whole accelerator complex must be done. From the detector side, upgrades of the existing Super-K detector are planned: the Hyper-K detector $(2 \times 500 \mathrm{kt}$ water Cherenkov detector in a side cavern at the Kamioka mine $)$ or a $100 \mathrm{kt}$ LArgon TPC detector located at the Okinoshima island at $658 \mathrm{~km}$ distance and $0.78 \mathrm{deg}$ offaxis. The immediate target of the $\mathrm{T} 2 \mathrm{~K}$ program is the confirmation of the present $\theta_{13}$ measurement and the the planned upgrades further continue to probe the CP violation in the neutrino sector [2].

For the neutrino beams in U.S.A, the future plans start with an upgrade of the NuMI facility to operate with a $700 \mathrm{~kW}$ beam that can be produced profiting from the $8 \mathrm{GeV}$ Recycler ring that became available after the Tevatron closure. The target and horn components of the NuMI beam must be redesigned to cope with the increased beam intensity of $4.910^{13}$ protons and shorter $1.33 \mathrm{sec}$ cycle. The upgraded facility will serve the new NOvA Neutrino Experiment located 


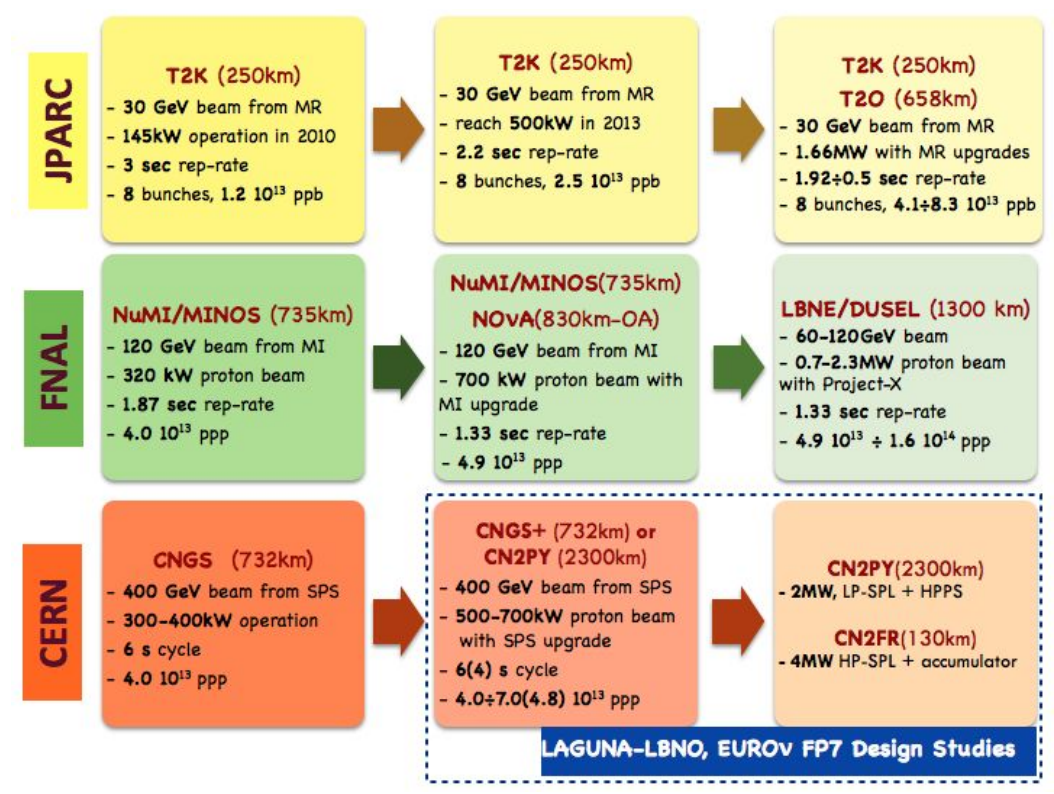

Figure 2: Road map for the conventional neutrino beam facilities.

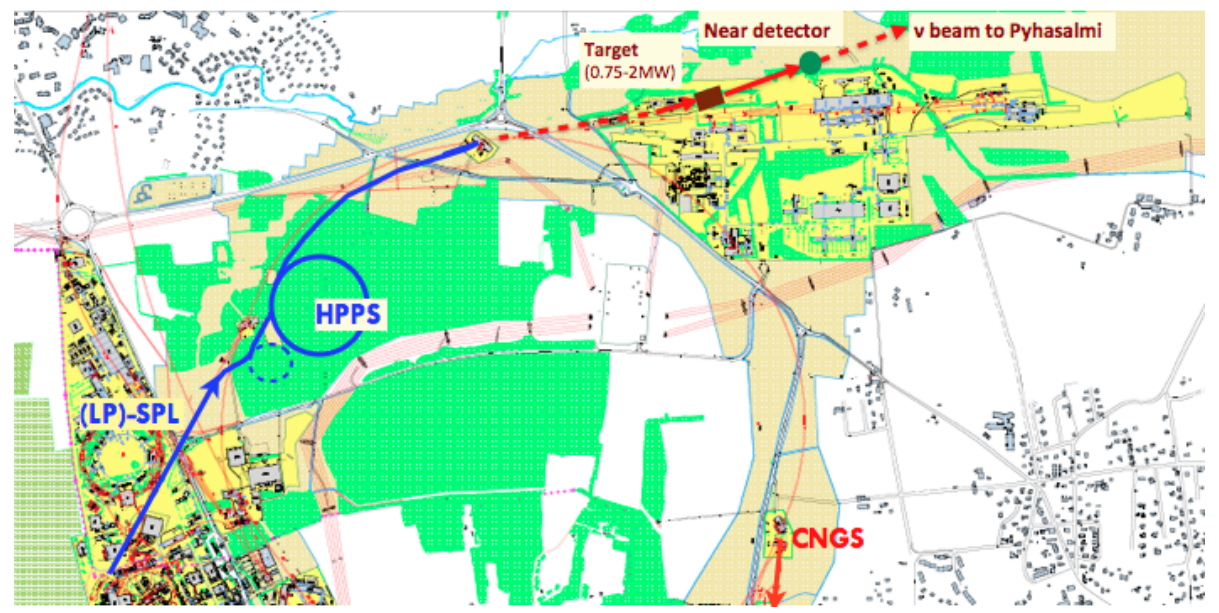

Figure 3: Schematic view of the CN2PY beam from CERN to Pyhasalmi mine in Finland, under study in the LAGUNA-LBNO/FP7 project [7].

14 miliradians off-axis at 810 kmdistance from FNAL in the north Minnesota close to the U.S.Canada border. In the second stage a new long-baseline neutrino beam (LBNE) is proposed, that sends an intense neutrino beam to large volume detectors at the Homestake mine in South Dakota, $1300 \mathrm{~km}$ distance from FNAL. The LBNE beam will require a new injector capable of delivering $\geq 2 \mathrm{MW}$ of protons in the $60-120 \mathrm{GeV}$ range, and its design must meet stringent technical and radiation safety requirements while minimizing beam and facility cost [3].

In Europe, an incremental approach for new long-baseline neutrino beams from CERN is under study within the LAGUNA-LBNO EU/FP7 project [7]. The new beam line (CERN to Pyhasalmi - CN2PY) will point North to a far detector located at the Pyhasalmi mine in Finland, at $2300 \mathrm{~km}$ distance from CERN. The large distance, offers unique physics opportunities for CP violation and 
neutrino mass hierarchy measurements. Initially a new beam line is constructed using the $400 \mathrm{GeV}$ beam from SPS like CNGS, with hopefully increased beam power up to $750 \mathrm{~kW}$ as a result of the machine upgrades for LHC (Figure 3). In a second phase, the beam power can be increased using the newly built LINAC4 followed by a LP-SPL to deliver a beam in a new high-power PS that can feed the CN2PY beam with a $\leq 2 \mathrm{MW}$ at $30-50 \mathrm{GeV}$. The long $2300 \mathrm{~km}$ distance to Pyhasalmi is also "magic distances" for a Neutrino Factory, i.e. with optimized sensitivity to the CP-violation and mass hierarchy parameters.

In parallel to te conventional beams, design studies for a Neutrino Factory, Super-beam and $\beta$ Beam options are ongoing, supported by the EUROnu/FP7 project [8]. The Super-beam design is similar to a conventional neutrino beam but for a high-power (4 MW) proton beam at high rep-rate $(50 \mathrm{~Hz})$ using the CERN HP-SPL coupled to an accumulator ring. The high-power and rep-rate impose extremely challenging conditions for the design and operation of the target and capture station. In the Super-beam option under study, the neutrino beam will point to a far water cherenkov detector located at the Frejus tunnel in France at $130 \mathrm{~km}$ distance from CERN. Synergy with a $\beta$ beam neutrino beam for $\gamma=100$ with $6 \mathrm{He} / 18 \mathrm{Ne} \beta$-emitter isotopes offer enhanced physics reach. The Neutrino Factory design is advancing through a global world-wide collaboration (IDS-NF). Significant progress has been achieved in addressing the critical R\%D issues for the facility like the target station, cooling channel and rapid acceleration all using proof-of-principle experiments. The Neutrino Factory remains the design choice for a facility with wider physics reach with sufficient performance to do precision measurements that will be needed to fully understand the physics behind the neutrinos.

\section{Aknowledgements}

I would like to acknowledge inputs and assistance from many colleagues working on the neutrino beams. The progress and achievements in designing and operating present and future neutrino facilities is only possible thanks to the motivation, hard work and collaboration of all.

\section{References}

[1] K. Nakamura et al. (Particle Data Group), J. Phys. G37, 075021 (2010).

[2] T2K web page: http://www.t2k.org

K. Abe et al. (T2K Collaboration), Phys. Rev. Lett. 107 (2011),041801

H. Kakuno, Presentation at NUFACT11 - CERN, Geneva Switzerland, http://cern.ch/nufact11

[3] NuMI Web page: http://www-numi.fnal.gov S. Childress, J. Strait, Presentation at NUFACT11 - CERN, Geneva Switzerland, http://cern.ch/nufact11.

[4] CNGS Web page: http://cern.ch/cngs

[5] N. Agafonova et al. (OPERA Collaboration), Phys. Lett. B691 (2010) 138

[6] C. Rubbia et al. (ICARUS Collaboration), 2011 JINST 6 P07011.

[7] LAGUNA, LAGUNA-LBNO Web page: http://laguna.ethz.ch:8080/Plone A. Rubbia, Presentation at NUFACT11 - CERN,Geneva Switzerland, http://cern.ch/nufact11

[8] EUROnu Web page: http://www.euronu.org 